\title{
Second-harmonic generation imaging analysis can help distinguish sarcoidosis from tuberculoid leprosy
}

Fabiane Leonel Utino

Marina Garcia

Paulo Eduardo Neves Ferreira Velho

Andréa Fernandes Eloy da Costa França

Rafael Fantelli Stelini

Vitor Bianchin Pelegati

Carlos Lenz Cesar

Elemir Macedo de Souza

Maria Letícia Cintra

Gislaine Vieira Damiani 


\title{
Second-harmonic generation imaging analysis can help distinguish sarcoidosis from tuberculoid leprosy
}

\author{
Fabiane Leonel Utino, ${ }^{\mathrm{a}, \mathrm{b}, *}$ Marina Garcia, ${ }^{\mathrm{a}}$ Paulo Eduardo Neves Ferreira Velho, ${ }^{\mathrm{b}}$ \\ Andréa Fernandes Eloy da Costa França, ${ }^{b}$ Rafael Fantelli Stelini, ${ }^{a}$ Vitor Bianchin Pelegati, ${ }^{c, d}$ \\ Carlos Lenz Cesar, ${ }^{\text {,d,e }}$ Elemir Macedo de Souza, ${ }^{\mathrm{b}}$ Maria Letícia Cintra, ${ }^{\mathrm{a}}$ and Gislaine Vieira Damiani ${ }^{\mathrm{c}, \mathrm{f}}$ \\ aUniversity of Campinas, Department of Pathology, Campinas, Brazil \\ bUniversity of Campinas, Department of Dermatology, Campinas, Brazil \\ 'Technology on Photonics Applied to Cell Biology, Campinas, Brazil \\ dUniversity of Campinas, "Gleb Wataghin" Institute of Physics, Campinas, Brazil \\ eFederal University of Ceará, Department of Physics, Fortaleza, Brazil \\ ${ }^{f}$ Federal Institute of Education, Science and Technology, São Paulo, Brazil
}

\begin{abstract}
Sarcoidosis and tuberculoid leprosy (TL) are prototypes of granulomatous inflammation in dermatology, which embody one of the histopathology limitations in distinguishing some diseases. Recent advances in the use of nonlinear optical microscopy in skin have enabled techniques, such as second-harmonic generation (SHG), to become powerful tools to study the physical and biochemical properties of skin. We use SHG images to analyze the collagen network, to distinguish differences between sarcoidosis and TL granulomas. SHG images obtained from skin biopsies of 33 patients with TL and 24 with sarcoidosis retrospectively were analyzed using first-order statistics (FOS) and second-order statistics, such as gray-level co-occurrence matrix (GLCM). Among the four parameters evaluated (optical density, entropy, contrast, and second angular moment), only contrast demonstrated statistical significance, being higher in sarcoidosis $(p=0.02 ; 4908.31$ versus 2822.17). The results may indicate insufficient differentiating power for most tested FOS and GLCM parameters in classifying sarcoidosis and TL granulomas, when used individually. But in combination with histopathology (H\&E and complementary stains, such as silver and fast acid stains), SHG analysis, like contrast, can contribute to distinguishing between these diseases. This study can provide a way to evaluate collagen distribution in granulomatous diseases. () The Authors. Published by SPIE under a Creative Commons Attribution 3.0 Unported License. Distribution or reproduction of this work in whole or in part requires full attribution of the original publication, including its DOI. [DOI: 10.1117/1.JBO.23.12.126001]

Keywords: granulomatous diseases; second-harmonic generation; sarcoidosis; tuberculoid leprosy.
\end{abstract}

Paper 180313RR received May 31, 2018; accepted for publication Nov. 7, 2018; published online Dec. 4, 2018.

\section{Introduction}

Leprosy is an infectious and contagious chronic disease, caused by Mycobacterium leprae, an obligate intracellular bacillus that mainly affects skin, nerves, and mucous membranes. But it can also affect the eyes, noses, joints, lymph node, internal organs, and bone marrow, especially in multibacillary patients. ${ }^{1,2}$ Currently worldwide, more than 200,000 new cases of leprosy are detected annually, with India, Brazil, and Indonesia accounting for $\sim 80 \%$ of all new cases. ${ }^{3}$ Brazil occupies second place in the world in absolute number of cases. In 2015, the detection rate was of $14.7 / 100,000$ inhabitants, with 28,761 new diagnosed cases. ${ }^{4}$ Unfortunately, even in endemic countries such as Brazil, leprosy diagnosis may be a major problem, with some areas presenting a mean delay in diagnosis of over two years. ${ }^{5,6}$ To make matters worse, in paucibacillary subtypes, such as tuberculoid forms of leprosy (TL) [characterized by tuberculoid granulomas on histology, Fig. 1(d)], bacilli are infrequently found, even with deep sections. Polymerase chain reaction is limited in M. leprae DNA detection in the tuberculoid pole of leprosy, ${ }^{7-9}$ and this type of mycobacterium cannot be cultivated in an artificial culture medium.

Not uncommonly, physicians have difficulty differentiating TL from sarcoidosis' cutaneous manifestation, because of

*Address all correspondence to: Fabiane Leonel Utino, E-mail: faby_utino@ yahoo.com.br their clinical and histological similarities. Sarcoidosis is an inflammatory disease of unknown etiology, characterized by sarcoidal or naked granulomas [Fig. 1(a)]. It mainly affects lungs, lymph nodes, and skin of people throughout the world, irrespective of age, gender, and race. The incidence averages from 3 to 50 cases per 100,000 among Europeans, 58 per 100,000 among West Indians, 14 per 100,000 among Asians, ${ }^{10}$ and 10 to 40 cases per 100,000 persons in the United States. ${ }^{11}$

TL should be treated with antibiotics association, whereas sarcoidosis with immunosuppression. Delays in initiating TL treatment may lead to disabling sequelae, which underscores the importance of distinguishing one from the other. Reticulin fibers are present in granulomas and make up a network that may be detected by silver stain. Their main component is type-III collagen, ${ }^{12,13}$ but they also contain fibronectin, types I and V collagen, and the basement membrane components laminin and type-IV collagen. ${ }^{13}$ Because reticulin fibers are usually scarce and fragmented in TL, ${ }^{14}$ some authors hypothesize that the preservation of normal reticulin network within granulomas is consistent with sarcoidosis.

We previously studied ${ }^{15}$ the reticulin fibers density inside the granulomas in TL [Fig. 1(e)] and sarcoidosis [Fig. 1(b)]. The median surface density of reticulin fibers was significantly higher in sarcoidosis. Nonetheless, using logistic regression, this variable did not discriminate one from the other.

For most diseases, histologic examination is still the "gold standard" for nosological diagnosis. Recent advances in the 


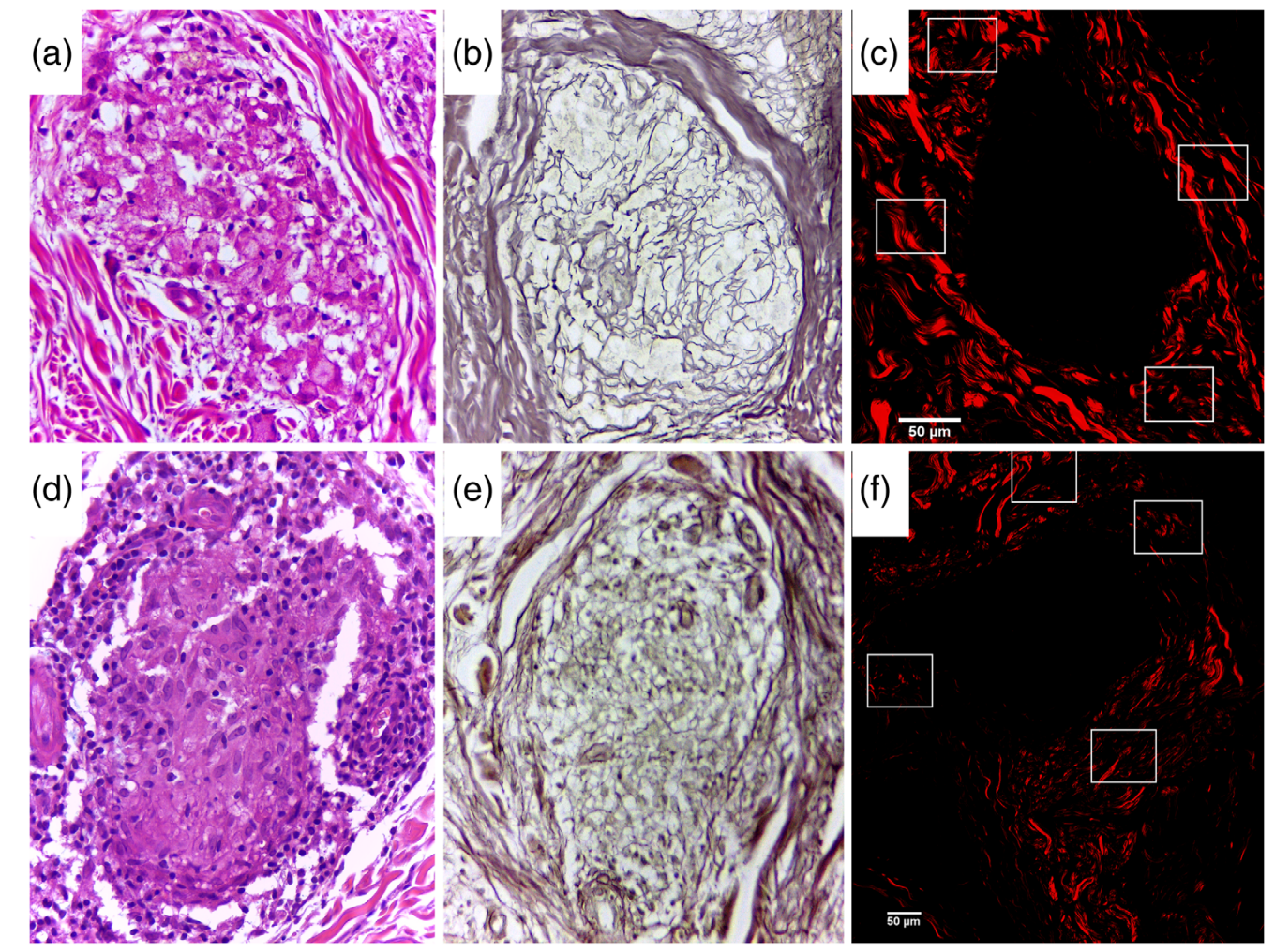

Fig. 1 Naked granuloma in sarcoidosis, with (a) peripheral fibrosis and (b) rich reticulin network. (d) Granuloma in tuberculoid leprosy, with a lymphocyte rim and (e) scanty reticulin network. (c) SHG images-granuloma in sarcoidosis and (f) tuberculoid leprosy. This figure contains four delimited (superior, inferior, right, and left) regions of interest (squares). There is a scant signal inside the granulomas; (a) and (d) H\&E and (b) and (e) silver (Gomori) stains. Original magnification: 500x.

use of nonlinear optical microscopy (NLOM) in skin samples have enabled techniques, such as second-harmonic generation (SHG) to become powerful tools for studying the physical and biochemical properties of skin. ${ }^{16}$ Utilizing SHG, phase matching of photons in noncentrosymmetric structures results in a scattering event in which two photons are combined into one photon at twice the frequency of the incident light. ${ }^{17}$ SHG has been proven an ideal noninvasive tool for examining collagen microstructure, cellularity, and crosslink content in gels in both in-vitro models and biological tissue. ${ }^{18}$ In animals, the collagen family represents the most abundant protein within the extracellular matrix. ${ }^{19}$ Fibrillary collagens have a noncentrosymmetric structure that can be imaged using NLOM. Recently, SHG has also been used for investigating collagen fiber orientation and structural changes in healthy tissues, such as human dermis, ${ }^{20,21}$ corneas, ${ }^{22}$ and within tumor microenvironment. ${ }^{23}$

Many studies have successfully extracted quantitative texture information, such as gray-level co-occurrence matrix (GLCM), ${ }^{24}$ from SHG images to evaluate collagen abnormalities. ${ }^{25-27}$ We were unable to find information on the value of SHG for the study of granulomatous diseases or for evaluating reticulin fibers, which are composed mainly of type-III collagen. In this study, we measured optical density and GLCM parameters of SHG images, using ImageJ program, to compare collagen networks in sarcoidosis and TL granulomas.

\section{Materials and Methods}

We obtained approval for this research project from the Institutional Review Board of the local Research Institutional
Ethics Committee (protocol \#651.612), prior to acquiring data. In this analytic observational study, we collected data from patients with TL and sarcoidosis, respectively, who were treated at the Dermatologic Outpatient Center of the Unicamp Hospital from 1996 to 2016. The study inclusion criteria required a combination of clinical, laboratorial, and histopathological examinations and the absence of other granulomatous diseases, in addition to a compatible clinical course after treatment. Thirty-three patients with TL and 24 patients with sarcoidosis fulfilled the inclusion criteria. Ex-vivo paraffin skin samples were $5 \mu \mathrm{m}$ sectioned and stained using the Hematoxilin and Eosin (H\&E) and Gomori methods.

\subsection{Image Acquisition by Nonlinear Optic Multiphoton Microscopy}

SHG images were utilized to evaluate collagen organization within and around granulomas. Images were acquired with an inverted Z.1 Axio Observer microscope equipped with a Zeiss LSM780 NLO confocal scanning head (Carl Zeiss AG, Jena, Germany), located at the National Institute of Photonics Applied to Cell Biology. Samples were excited by a Ti:Sapphire laser (MaiTai, Spectra Physics, Santa Clara, California) with emission at $800 \mathrm{~nm}$ of wavelength and $<100$-fs pulses, using $40 \times / 1.3$ numerical aperture (NA) objective. The laser system was equipped with an acousto-optic modulator (AOM) for laser power attenuation. We placed a broadband quarter wave plate (1/4-Newport) in the laser beam path, after the AOM and before the LSM780 scanning head, to have a circular polarized light to avoid anisotropies for different fibril's directions. 
A forward SHG signal was collected by the condenser lens (Carl Zeiss 0.55 NA-WD $26 \mathrm{~mm}$ ) and acquired by a nondescanned detector (NDD) that is part of the Zeiss system. To avoid laser light detection, a 690 short-pass filter was employed. A filter cube with a dichroic LP405-nm wavelength (Semrock Di02-R405-25x36) and a bandpass filter with center wavelength of 390/40 nm (Semrock FF01-390/40-25) were placed in front of the NDD detector to filter unwanted fluorescence light from the desired SHG signal. Calculated lateral and axial theoretical resolution were $\sim 280$ and $880 \mathrm{~nm}$. Average laser power was in the order of $10 \mathrm{~mW}$ at the sample, and all acquisition parameters remained equal in all images, with $512 \times 512$ pixels and $25.2 \mathrm{~ms} /$ pixel during acquisition for reducing unwanted noise. To obtain a complete image of the granuloma, we acquired tile scans $(512 \times 512)$ that were stitched in larger mosaics.

\subsection{Collagen Automatic Quantification}

Collagen analysis was blindly performed using ImageJ (v1.45) software. Each SHG image was converted into 256 levels of gray, which were manually selected from four regions of interest (ROIs), superior, inferior, right, and left [Figs. 1(c) and 1(f)] $1212 \mu \mathrm{m}^{2}$ within and around the granuloma (imageJ). Since granulomas usually have a rounded or ovoid shape, we standardized the sites of collagen analysis, in all cases, in four different ROIs, so that it would be possible to obtain a more representative mean value of each granuloma analysis, instead of choosing just one random ROI. In each box, we calculated the optical density and texture features from the GLCM [contrast, second angular moment (SAM), and entropy].

The collagen density in images was calculated using optical density (the product of area and mean gray value). Changes in collagen fibril morphology were tracked by texture analysis of SHG images using first-order statistics (FOS) and second-order statistics such as GLCM. FOSs are parameters extracted directly from the original image and do not consider neighboring values, while second-order statistics are derived from a matrix (e.g., GLCM) which is built upon interpixel correlation of the original image, which in turn depends on the spatial arrangements of pixel intensities present in ROI. ${ }^{28}$

\subsection{Statistical Analysis}

Exploratory analysis was done through summary measures (mean, standard deviation, minimum, median, and maximum). The groups were compared by the Mann-Whitney test; we adopted $5 \%$ as the significance level.

\section{Results}

We studied 33 patients with TL and 24 with sarcoidosis. Clinical and histological data have been previously reported. ${ }^{15}$

After SHG images acquisition, we concluded that a very scant signal could be detected inside the granulomas. Therefore, we decided to analyze solely the signal around them, choosing four ROIs per granuloma [Figs. 1(c) and 1(f)]. Subsequently, we used ImageJ software to quantify four parameters already established for collagen study (density, contrast, SAM, and entropy).

The collagen optical density was higher in sarcoidosis cases than in TL cases (Fig. 2), but the results were not significant $(p=0.06)$. Among the GLCM parameters, entropy and SAM values were similar, but contrast was significantly higher in sarcoidosis ( $p=0.02 ; 4908.31$ versus 2822.17).

\section{Discussion}

Sarcoidosis and TL are prototypes of granulomatous inflammation in dermatology and together they embody one of the histopathology limitations in distinguishing some diseases. Granulomatous inflammation is a distinctive pattern of chronic inflammation characterized by aggregates of activated macrophages that assume epithelioid appearance. ${ }^{29}$ These cells are firmly attached to one another, forming aggregates. Older granulomas may have a rim of fibroblasts and connective tissue. ${ }^{29}$ Histologically, in TL, epithelioid granulomas are well defined with an evident rim of lymphocytes that secrete cytokines, responsible for continuing macrophage activation. They classically distribute around the appendages and nerves. In sarcoidosis, noncaseating epithelioid-cell granulomas are found, with randomly distributed scant lymphocyte rims. Nonetheless, these findings are not truly helpful for differential diagnosis, in view of the number of reported sarcoidosis cases first treated as TL and vice versa. ${ }^{30-33}$ SHG imaging analysis has proven to be useful in determining the directionality and organization of the fibrillary collagen in the skin, in both health and disease. As far as we could ascertain, there are no English-language studies on granulomatous diseases using SHG.

The methods described to study extracellular matrix and tissue collagen organization on histological sections vary. Masson's trichrome and picrosirius red on bright field or polarization microscopy are most commonly used. ${ }^{34}$ However, these methods require multiple steps of tissue processing (while SHG image, even with some limitations, can be obtained from H\&Estained specimens), and such sample preparations can lead to undesirable morphological alterations in extracellular matrix. ${ }^{35}$ Furthermore, SHG signals arise from induced polarization rather than from absorption, leading to substantially reduced photobleaching and phototoxicity relative to fluorescence methods. ${ }^{36}$ Morphometry may be carried out with the aid of an eyepiece cycloids grid coupled with an ocular lens or through the use of virtual cycloids (ImageJ), but this is a time-consuming procedure. ${ }^{15}$ With the help of a computer and dedicated software tools, automatic quantification provides faster results on thickness and quantity of collagen fibers. On the other hand, SHG microscopy allows increased imaging penetration depths and enhanced axial depth discrimination images in the study of texture and orientation study of collagen. ${ }^{27}$ Additionally, since granulomatous inflammation can give rise to changes in the assembly of collagen and, consequently, constrain conventional optical microscopy, it is precisely the increase in collagen's overall asymmetry that allows SHG to be a direct sensor of supramolecular structure. For example, Nadiarnykh et al. ${ }^{37}$ have demonstrated that SHG can reveal differences in the collagen morphology unable to be detected by other optical methods, when analyzing connective tissue disorder "Osteogenesis Imperfecta," characterized by abnormal collagen assembly. Therefore, SHG imaging is an attractive alternative to conventional histology for studying tissue composition and visualizing the molecular structure of collagen due to its label-free nature, high sensitivity, and specificity. ${ }^{38,39}$

The amount of signal produced by many sources of SHG is dependent on the polarization state of the incident laser light, relative to scattering due to the molecular structure. ${ }^{40,41}$ This nonlinear process is also sensitive to phase-matching properties, ${ }^{36}$ local anisotropy, and distribution of molecular hyperpolarizabilities, ${ }^{42}$ and it depends on the angle between the collagen fiber and the imaging plane. Moreover, in a collagenous tissue, 

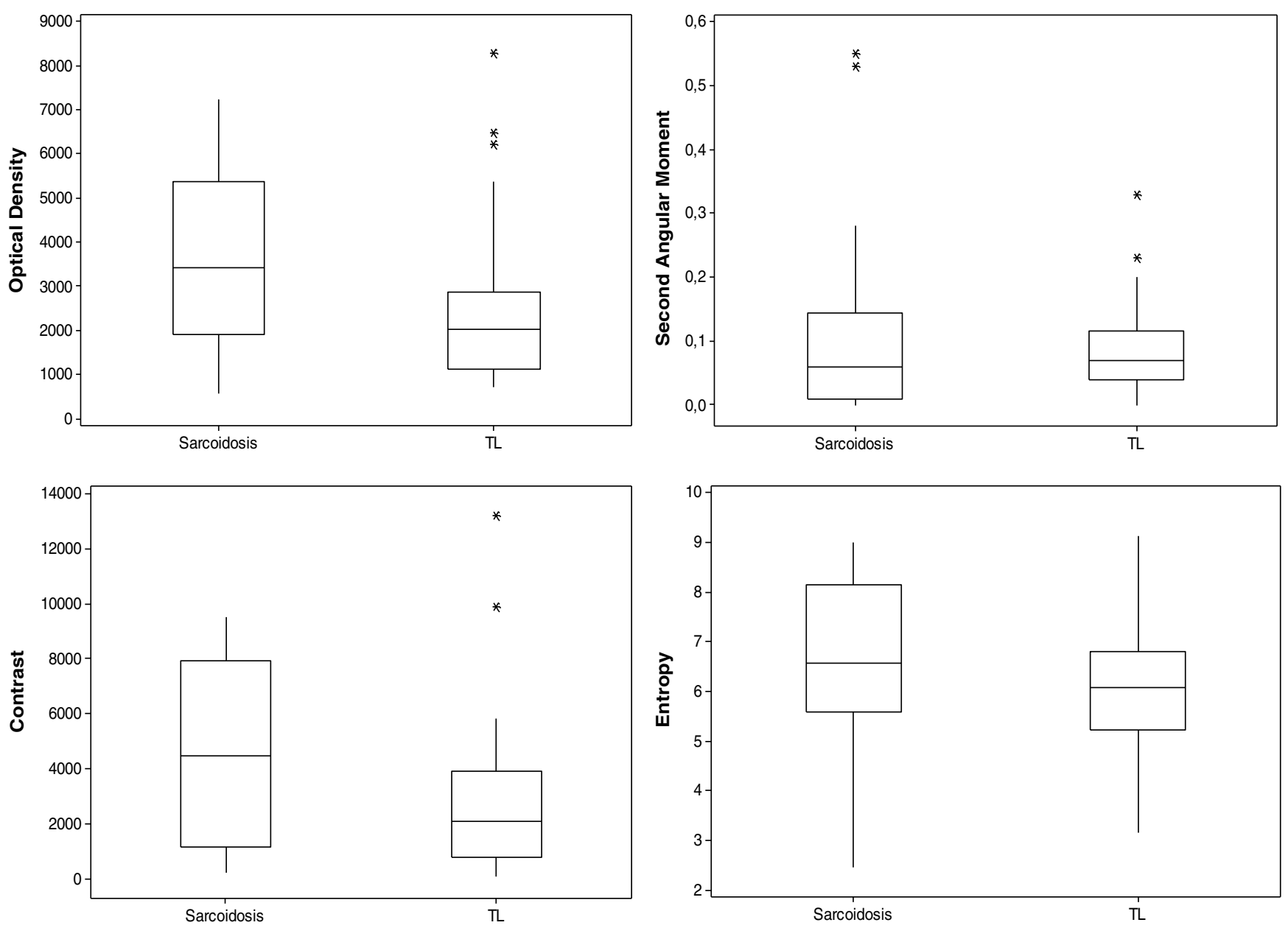

Fig. 2 Comparison of optical density and texture parameters (SAM, contrast, and entropy) calculated for sarcoidosis and TL group defined in the text. The top and bottom of each rectangular box denote the 75th and 25th percentiles, respectively, with the median shown inside the box. Vertical bars extending from each box represent the 90th and 10th percentiles. After Mann-Whitney test, only contrast presented statistical significance among the four parameters $(p=0.02)$.

the overall signal is predominantly propagated in the forward direction, compared to an isolated individual fiber that may radiate SHG signal both forward and backward. ${ }^{18}$ To excite all fiber orientations equally, we used quarter wave plate to generate a circular polarized beam that can generate a uniform excitation in all fiber directions (both parallel or perpendicular collagen fibers), and enabling detection of the signal generated from all fibers within the tissue, independent of their orientation.

SHG imaging has already been used in the analysis of fresh biopsies, cryopreserved tissues, in-vivo and H\&E-stained specimens. In contrast to hematoxylin, eosin accumulates in the cytoplasm and extracellular matrix, and has been reported to contribute to emission signaling in fluorescence lifetime imaging microscopy and multiphoton microscopy. ${ }^{43}$ In a study where the acquisition of nonlinear images from $\mathrm{H} \& \mathrm{E}$-stained sections before and after a de-staining protocol was evaluated by Monaghan et al., ${ }^{44}$ it was concluded that, although the multiphoton emission signals were significantly increased, most likely due to the presence of residual eosin in the tissue sections, SHG signaling was not significantly affected by the histological dyes after all. It is important to point out that multiphoton microscopy and irradiation with ultrashort lasers can produce physical damage, especially photodamage in unstained cells. ${ }^{45}$
In addition, Galli et al. $^{46}$ concluded that the fluorescence increase induced by photodamage is a process common to in vivo, fresh, and ex-vivo tissue. Although long acquisition times can possibly impact on photodamage, since it is a comparative study, a standardization of the parameters for both groups could minimize this limitation.

Originally, we aimed to compare (1) SHG signal measurement inside the granuloma, to evaluate the reticulin fibers density and texture (examined in our first study through morphometry in silver-stained sections), since reticulin fibers are mainly composed of type-III collagen, and (2) collagen fiber density and texture around granulomas. However, we found that SHG signal inside granulomas was very tenuous and sometimes even absent [Figs. 1(c) and 1(f)]. This is because reticulin fibers are also composed of other elements, such as fibronectin and laminin, which may not be detected by SHG. Also, since nonfibrillary collagens do not form centrosymmetric structures, and therefore, cannot be demonstrated by $\mathrm{SHG}^{18}{ }^{18}$ we envisage that collagen may experience modifications in its fibrillary structure inside granulomas. Accordingly, we evaluated SHG signal around each granuloma in four ROIs (superior, inferior, right, and left), in three different images per sample. The final data corresponded to the mean of all these values. 
Many SHG imaging studies solely describe collagen organization, but we believe it is important to seek correlations between SHG images and pathology by means of quantifiable measures for objective comparison. One possibility is to quantify image intensity by pixel-counting techniques or by measuring image texture from GLCM using ImageJ software. The mean density provides measures of the overall lightness/ darkness of the image and can be associated to the amount of collagen fibers, which is proportional to the detected SHG signal. ${ }^{18}$ Since SHG signal also depends on the thickness of nonlinear medium, and this may complicate interpretation of mean density, all ex-vivo paraffin skin samples were $5 \mu \mathrm{m}$ sectioned and stained using the H\&E.

GLCM analysis is a widely used texture analysis method developed by Haralik et al. ${ }^{24}$ GLCM is performed by counting the number of occurrences of a gray level adjacent to another gray level, at a specified pixel distance and direction. The result is a matrix with rows and columns representing gray levels and elements containing the probability of the gray-level co-occurrence. High contrast occurs when an image has a high number of pixel pairs with large differences in gray level occurring at the specified separation and orientation. Entropy is a textural parameter and has been associated with the degree of fiber organization, and therefore, can be very useful for the characterization of several types of tissues and conditions. ${ }^{47}$ SAM is highest in images with uniform gray level or uniform gray-level differences at the specified separation and lower for those with more variation in gray levels.

Analogous to the reticular fibers density, we expected to find a higher collagen density in sarcoidosis cases. In fact, the mean density in the latter was superior (3523.59 versus 2459.59 ), but the values were not significant $(p=0.06)$, perhaps because of limited sample size.

Entropy measures the lack of spatial organization inside the computational window. Entropy is usually high in a rough texture and low when the texture is more homogeneous or smooth. ${ }^{35}$ We expected a lower entropy value in sarcoidosis cases supported by the presence of thicker and better-defined collagen fibers inside and around their granulomas compared to the TLs. However, the mean entropies of TL (6.17) and sarcoidosis $(6.51)$ were similar $(p=0.34)$.

The energy feature is sometimes referred to as the SAM or uniformity of GLCM. The lowest value of energy is attained when there are no dominant gray levels. Most gray levels are equally probable. It is expected that GLCM SAM would change for different collagen morphologies because collagen width and spacing affect the transition of gray levels across the image. ${ }^{35}$ We thought we would obtain a lower average of SAM in TL cases because their fibers are thin and fragmented, causing higher variation in gray levels when moving across the image. We indeed had a lower SAM mean in TL (0.09) than in sarcoidosis (0.12) but without statistical significance.

Among the four parameters analyzed, only contrast presented statistical significance $(p=0.02)$, higher in sarcoidosis than in TL (Fig. 2), to wit: in sarcoidosis, collagen around granulomas presented a greater number of pixel pairs with large differences in gray level. A previous study ${ }^{15}$ demonstrated that the presence of fibrosis in skin dermis is a predictive histologic finding for sarcoidosis diagnosis instead of TL. Because of the higher fibrosis around granulomas in sarcoidosis, the collagen fibers surrounding them may be thicker and more irregular in shape, size, and length, presenting a more heterogeneous gray-level acquisition. In addition, in TL cases [Fig. 1(e)], reticular fibers appeared to be thinner and in less quantity around granulomas, when compared to sarcoidosis [Fig. 1(b)]. Since SHG weakly captured reticulin fibers, perhaps higher reticulin fibers density between thicker collagen fibers in sarcoidosis is a factor which contributes to higher contrast in GLCM analysis.

In summary, these results indicate insufficient differentiating power for most tested FOS and GLCM parameters in distinguishing sarcoidosis from TL granulomas, when used individually. But in combination with histopathology (H\&E and complementary stains, such as silver and fast acid stains), contrast parameter of SHG analysis can be an additional element to enhance the summation of features that may be required to assist in disease distinction. In the initial stages, this method provides a better understanding of the changes in the distribution of collagen around the granulomas of both diseases. Finally, this study may provide a method of evaluating collagen distribution not only for sarcoidosis or TL, but also in other granulomatous diseases.

\section{Conclusion}

SHG microscopy is a powerful tool for examining biological tissue. In this study, we highlighted the capability of nonlinear microscopy for dermatological imaging, which helps to distinguish two diseases that sometimes appear identical when using traditional linear optical microscopy examination. In a clinical setting, SHG has been used to evaluate collagen structure, in conjunction with in-vivo noninvasive tools, among a number of dermatological diseases. Perhaps in the near future, this may also be useful for other granulomatous diseases.

\section{Disclosures}

The authors have no relevant financial interests in this article and no potential conflicts of interest to disclose.

\section{Acknowledgments}

This study was supported by the São Paulo Research Foundation-FAPESP (Grant Nos. 2016/22927-7, 2017/ 17149-8, and 11/51959-0) and by Coordination of Superior Level Staff Improvement (CAPES) (Grant No. 01-P-3368/ 2017). We would like to express our thanks for the access to equipment and assistance provided by the National Institute of Science and Technology on Photonics Applied to Cell Biology (INFABIC), which is cofounded by FAPESP (Grant No. 2014/50938-8) and by National Council for Scientific and Technological Development (CNPq) (Grant Nos. 465699/ 2014-6 and 312049/2014-5). We are also grateful to Adilson A. Piaza and Mercedes F. Santos for assistance in photographic documentation, Ana C. Piaza and Luzia A. Magalhães for technical assistance, and Cleide A.M. Silva and Juliana L.P. Argenton for statistical assistance.

\section{References}

1. R. E. Pfaltzagraff and A. Bryceson, "Leprosy," Clinical Leprosy, 2nd ed., pp. 15-30, Churchill Livingstone, New York, Edinburg (1979).

2. S. Talhari et al., "Aspectos Gerais da Hanseníase, Agente Etiológico, Transmissão, Patogenia, Classificação, Manifestação Clínica, Diagnóstico," in Hanseníase, S. Talhari et al., Eds., 5th ed., pp. 94172, Di Livros, Rio de Janeiro, Brazil (2015).

3. Ministry of Health and WHO, "Global leprosy update 2014: need for early case detection," Wkly. Epidemiol. Rec. 90(36), 461-474 (2015). 
4. Ministry of Health, "Registro ativo: número e percentual, Casos novos de hanseníase: número, coeficiente e percentual, faixa etária, classificação operacional, sexo, grau de incapacidade, contatos examinados, por estados e regiões," Brazil, http://portalsaude.saude.gov.br/ images/pdf/2015/julho/27/Dados-2014?-final.pdf (7 April 2016).

5. A. S. Lima et al., "Leprosy in a University Hospital in Southern Brazil," An. Bras. Dermatol. 90(5), 654-659 (2015).

6. P. D. Deps et al., "Delay in the diagnosis of leprosy in the metropolitan region of Vitoria, Brazil," Lepr. Rev. 77(1), 41-47 (2006).

7. I. M. Goulart et al., "Detection of Mycobacterium leprae DNA in skin lesions of leprosy patients by PCR may be affected by amplicon size," Arch Dermatol. Res. 299(5-6), 267-271 (2007).

8. D. L. Williams et al., "The use of a specific DNA probe and polymerase chain reaction for the detection of Mycobacterium leprae," J. Infect Dis. 162(1), 193-200 (1990).

9. K. Kampirapap et al., "DNA amplification for detection of leprosy and assessment of efficacy of leprosy chemotherapy," Int. J. Lepr. Other Mycobact. Dis. 66(1), 16-21 (1998).

10. M. W. McNicol and P. J. Luce, "Sarcoidosis in a racially mixed community," J. R. Coll. Physicians London 19(3), 179-183 (1985).

11. Y. Hosoda, S. Sasagawa, and N. Yasuda, "Epidemiology of sarcoidosis: new frontiers to explore," Curr. Opin. Pulm. Med. 8, 424-428 (2002).

12. H. Konomi, J. Sano, and Y. Nagai, "Immunohistological localization of types I, III, and IV (basement membrane) collagens in the lymph node: co-distribution of types I and III collagens in the reticular fibers," Biomed. Res. 2, 536-545 (1981).

13. T. L. McCurley et al., "The extracellular matrix in 'sclerosing' follicular center cell lymphomas: an immunohistochemical and ultrastructural study," Hum. Pathol. 17, 930-938 (1986).

14. V. Nirmala, C. J. Chacko, and C. K. Job, "Tuberculoid leprosy and tuberculosis skin-a comparative histopathological study," Lepr. India 49(1), 65-69 (1977).

15. F. L. Utino et al., "Histomorphometric approach to differentiate skin lesions of tuberculoid leprosy from sarcoidosis," J. Cutan. Pathol. 45(2), 111-117 (2018).

16. K. M. Hanson and C. J. Bardeen, "Application of nonlinear optical microscopy for imaging skin," Photochem. Photobiol. 85(1), 33-44 (2009).

17. P. J. Campagnola et al., "High-resolution nonlinear optical imaging of live cells by second harmonic generation," Biophys. J. 77, 3341-3349 (1999).

18. L. Mostaço-Guidolin, N. L. Rosin, and T. L. Hackett, "Imaging collagen in scar tissue: developments in second harmonic generation microscopy for biomedical applications," Int. J. Mol. Sci. 18(8), 1772 (2017).

19. M. K. Gordon and R. A. Hahn, "Collagens," Cell Tissue Res. 339(1), 247-257 (2010)

20. R. Cicchi et al., "Scoring of collagen organization in healthy and diseased human dermis by multiphoton microscopy," J. Biophotonics 3(1-2), 34-43 (2010).

21. T. Yasui, Y. Tohno, and T. Araki, "Characterization of collagen orientation in human dermis by two-dimensional second-harmonicgeneration polarimetry," J. Biomed. Opt. 9(2), 259-264 (2004).

22. M. Han, G. Giese, and J. F. Bille, "Second harmonic generation imaging of collagen fibrils in cornea and sciera," Opt. Express 13(15), 57915797 (2005).

23. E. Brown et al., "Dynamic imaging of collagen and its modulation in tumors in vivo using second harmonic generation," Nat. Med. 9(6), 796800 (2003).

24. R. M. Haralick, K. Shanmugam, and I. Dinstein, "Textural features for image classification," IEEE Trans. Syst. Man Cybern. SMC-3, 610-621 (1973).

25. J. Adur et al., "The severity of Osteogenesis imperfecta and type I collagen pattern in human skin as determined by nonlinear microscopy: proof of principle of a diagnostic method," PLoS One 8(7), e69186 (2013).

26. K. Tilbury and J. Campagnola, "Applications of second-harmonic generation imaging microscopy in ovarian and breast cancer," Perspect. Medicin. Chem. 7, 21-32 (2015).

27. G. V. Damiani et al., "Idiopathic atrophoderma of Pasini and Pierini: a case study of collagen and elastin texture by multiphoton microscopy," J. Am. Acad. Dermatol. 77(5), 930-937 (2017).
28. M. Bevk and I. Kononenko, "A statistical approach to texture description of medical images: a preliminary study," in Proc. of the 15th IEEE Symp. on Computer-Based Medical Systems, IEEE, Vol. 64, pp. 239244 (2002).

29. V. Kumar et al., Robbins Basic Pathology, 8th ed., Saunders/Elsevier, Philadelphia (2007).

30. A. E. Burdick et al., "Hansen's disease in a patient with a history of sarcoidosis," Int. J. Lepr. Other Mycobact. Dis. 68(3), 307-311 (2000).

31. S. Kaur et al., "Cutaneous sarcoidosis masquerading as relapsed borderline tuberculoid leprosy?" Int. J. Lepr. Other Mycobact. Dis. 61(3), 455-458 (1993).

32. K. Singh et al., "Sarcoidosis masquerading as leprosy, pulmonary tuberculosis and urolithiasis," J. Assoc. Physicians India 38(9), 657-659 (1990).

33. K. Ramanujam, "Tuberculoid leprosy or sarcoidosis? A diagnostic dilemma," Lepr. India 54(2), 318-323 (1982).

34. P. Whittaker et al., "Quantitative assessment of myocardial collagen with picrosirius red staining and circularly polarized light," Basic. Res. Cardiol. 89, 397-410 (1994).

35. L. B. Mostaço-Guidolin et al., "Collagen morphology and texture analysis: from statistics to classification," Sci. Rep. 3, 2190 (2013).

36. R. LaComb et al., "Phase matching considerations in second harmonic generation from tissues: effects on emission directionality, conversion efficiency and observed morphology," Opt. Commun. 281(7), 18231832 (2008).

37. O. Nadiarnykh et al., "Second harmonic generation imaging microscopy studies of osteogenesis imperfecta," J. Biomed. Opt. 12(5), 051805 (2007).

38. P. Campagnola, "Second harmonic generation imaging microscopy: applications to diseases diagnostics," Anal. Chem. 83, 3224-3231 (2011).

39. C. P. Pfeffer et al., "Multimodal nonlinear optical imaging of collagen arrays," J. Struct. Biol. 164, 140-145 (2008).

40. R. M. Williams, W. R. Zipfel, and W. W. Webb, "Multiphoto nmicroscopy in biological research," Curr. Opin. Chem. Biol. 5, 603-608 (2001).

41. W. R. Zipfel, R. M. Williams, and W. W. Webb, "Nonlinear magic: multiphoton microscopy in the biosciences," Nat. Biotechnol. 21, 1369-1377 (2003).

42. I. Freund and M. Deutsch, "Second-harmonic microscopy of biological tissue," Opt. Lett. 11(2), 94-96 (1986).

43. J. Gu et al., "Quantitative diagnosis of cervical neoplasia using fluorescence lifetime imaging on haematoxylin and eosin stained tissue sections," J. Biophotonics 7(483), 1-9 (2014).

44. M. G. Monaghan et al., "Enabling multiphoton and second harmonic generation imaging in paraffin-embedded and histologically stained sections," Tissue Eng. Part C Methods 22(6), 517-523 (2016).

45. F. Fischer et al., "Risk estimation of skin damage due to ultrashort pulsed, focused near-infrared laser irradiation at $800 \mathrm{~nm}$," J. Biomed. Opt. 13(4), 041320 (2008).

46. R. Galli et al., "Intrinsic indicator of photodamage during label-free multiphoton microscopy of cells and tissues," PLoS One 9(10), e110295 (2014).

47. L. B. Mostaço-Guidolin et al., "Evaluation of texture parameters for the quantitative description of multimodal nonlinear optical images from atherosclerotic rabbit arteries," Phys. Med. Biol. 56, 5319-5334 (2011).

Fabiane Leonel Utino received her MD in 2011, dermatology residency degrees in 2015, and her PhD in medical science from the University of Campinas (Unicamp) Medical School in 2018. Her research specialization is dermatopathology.

Marina Garcia is a medical student at Pontifical Catholic Unicamp. In 2017, she received a one-year research scholarship from the Pathology Department at Unicamp. Dermatology is her major field of interest. She aims to specialize in this area in a postgraduate program.

Paulo Eduardo Neves Ferreira Velho received his MD degree in 1990 and medical sciences PhD in 2001 from the Unicamp Medical School, where he currently serves as an associate professor. His area of research is in medicine, with an emphasis on infectious diseases 
and dermatology; his focus is in the following areas: skin, health education, dermatology, and human bartonelloses.

Andréa Fernandes Eloy da Costa França graduated from the Federal University of Ceará in 2000 and received her $\mathrm{PhD}$ in internal medicine at the Unicamp in 2011. She is a dermatologist and professor in the Internal Medicine Department at the Unicamp. Her research areas include leprosy, autoimmune diseases, and allergies.

Rafael Fantelli Stelini received his medical training at the Pontifical Catholic Unicamp, Brazil. He completed his residency at the Unicamp, Brazil, and was approved as an anatomic pathologist in 2008. Currently, he is an assistant physician in the Department of Pathological Anatomy of the Hospital of Clinics of Unicamp, working mainly in the Dermatopathology Division.

Vitor Bianchin Pelegati received his bachelor's degree in physics from the Unicamp in 2006, his master's degree in 2010, and PhD in physics on the subject of optical microscopies of coherent process in 2016. He is a technician at the National Institute of Photonics Applied to Cell Biology. His research interests are multiphoton microscopies and coherent Raman.

Carlos Lenz Cesar received his degree in physics from the Federal University of Ceará in 1977, his master's degree in 1979, and his PhD in physics in 1985 from the Unicamp. Currently, he is a full professor at the Institute of Physics Gleb Wataghin in Unicamp. His research interests are integrated biophotonics microscopy such as TPEF, CARS, FLIM, FRET, Raman, hyper-Raman, and micromanipulations using optical tweezers, laser cutting and AFM/tip enhancement, and quantum dots physics and applications.

Elemir Macedo de Souza received his degree in medicine from Pontifical Catholic University of São Paulo, and his PhD in dermatology from the Unicamp. Currently, he is serving as a professor of dermatology in the Clinical Department, Medical School, Unicamp. His research interests are dermatopathology and cutaneous manifestations of systemic disease.

Maria Letícia Cintra received her degree in medicine from University of São Paulo (USP) and her PhD in pathology from the State Unicamp. Currently, she is a full professor in the Pathology Department of Unicamp Medical School. Her research interest is dermatopathology.

Gislaine Vieira Damiani received her bachelor's degree in biological sciences from the University of Americana in 2007, and her master's degree in 2009 and $\mathrm{PhD}$ in medical pathophysiology in 2012 from the Unicamp. Currently, she is a full professor at the Federal Institute of Education, Science and Technology of São Paulo. Her research interests are extracellular matrix and bartonelosis. 